\title{
UBE2T promotes proliferation and regulates PI3K/Akt signaling in renal cell carcinoma
}

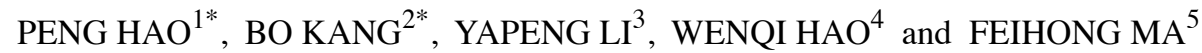 \\ ${ }^{1}$ Department of Urology Surgery, First Affiliated Hospital of Jiamusi University, Jiamusi, Heilongjiang 154003; \\ ${ }^{2}$ Department of Central Sterile Supply, First Affiliated Hospital of Harbin Medical University, Harbin, Heilongjiang 150001; \\ ${ }^{3}$ Department of Medical Record; ${ }^{4}$ Distinguished Physician Class; ${ }^{5}$ Department of Interventional Radiology, \\ First Affiliated Hospital of Jiamusi University, Jiamusi, Heilongjiang 154003, P.R. China
}

Received August 13, 2018; Accepted March 25, 2019

DOI: $10.3892 / \mathrm{mmr} .2019 .10322$

\begin{abstract}
Renal cell carcinoma (RCC) is a common malignant tumor globally. The overall survival of patients with $\mathrm{RCC}$ is poor; one important factor is tumor heterogeneity. Ubiquitin-conjugating enzyme E2T (UBE2T) has been reported to act as an oncogene in various types of cancer; however, its role in RCC has yet to be investigated. In the present study, UBE2T was demonstrated via reverse transcription-quantitative PCR analysis to be significantly upregulated in RCC samples and cell lines compared with in normal tissue and cells. Additionally, UBE2T expression was significantly associated with late tumor stage and high grade in patients with RCC, and patients with high UBE2T expression exhibited poor prognosis compared with patients with low expression. Following knockdown of UBE2T in 786-O cells using RNA interference technology, the proliferation and colony formation of cells were inhibited as determined by an MTT assay and crystal violet staining, respectively; however, the migration and invasion of 786-O cells were not affected, as determined by wound-healing assay and Transwell assays, respectively. Xenograft RCC tumor growth in vivo was also significantly suppressed. The expression levels of two mesenchymal cell markers, N-cadherin and vimentin, were reduced following UBE2T knockdown, whereas E-cadherin and fibronectin levels were increased as determined by western blotting, indicating that epithelial-mesenchymal transition was suppressed. In addition, the phosphorylation levels of PI3K, Akt and mTOR were notably decreased following UBE2T knockdown, but were increased when UBE2T was overexpressed. Wortmannin,
\end{abstract}

Correspondence to: Professor Feihong Ma, Department of Interventional Radiology, First Affiliated Hospital of Jiamusi University, 348 Dexiang Road, Jiamusi, Heilongjiang 154003, P.R. China E-mail:mfh0220@126.com

*Contributed equally

Key words: ubiquitin-conjugating enzyme E2T, PI3K, Akt, mTOR, renal cell carcinoma an Akt inhibitor, reversed the UBE2T overexpression-induced increase in the phosphorylation of PI3K, Akt and mTOR. Similarly, the UBE2T overexpression-induced promotion of 786-O cell proliferation was also attenuated by wortmannin. In conclusion, UBE2T promoted the proliferation of RCC cells by regulating PI3K/Akt signaling, suggesting it may be a novel target for the treatment of patients with RCC.

\section{Introduction}

Renal cell carcinoma (RCC) is among the top 10 most lethal types of cancer and is the cause of $>10,000$ cases of mortality every year (1). According to the most recent statistics published by the American Cancer Society, 65,340 individuals were predicted to be diagnosed with RCC in 2018, whereas the estimated mortality rate was $\sim 14,970$ cases (1). Patients with RCC frequently encounter recurrence and/or metastasis in late stages, leading to poor clinical outcome despite the improved treatments for RCC currently in use $(2,3)$. The 5-year survival rate for patients with kidney cancer with distant metastasis is only $12 \%$, whereas it is $67 \%$ for patients with localized cancer (1). At present, $\sim 16 \%$ of patients are diagnosed with distant metastasis (1); however, there is no agreement regarding the molecular mechanisms underlying RCC. Next-generation sequencing technology has identified dozens of gene mutations associated with various type of cancer; however, only mutations of certain driver genes serve dominant roles in the development and progression of cancer (4). A number of genes associated with RCC have been identified, but the heterogeneity of the condition increases the importance of identifying novel genes and further understanding the molecular mechanisms underlying RCC $(5,6)$.

Ubiquitin-conjugating enzyme E2T (UBE2T) is a member of the ubiquitin-proteasome family; this family is an efficient protein-modification system that serves important roles in almost all cell behaviors, including DNA replication, cell proliferation, differentiation, apoptosis and angiogenesis $(7,8)$. UBE2T has been reported to bind to FA complementation group L protein via its UBC domain, promoting the formation of the Fanconi anemia (FA) core complex and activating the FA signaling pathway (9-11). In addition, UBE2T has been reported to cause FA, increasing the risk of acute 
myeloid leukemia or head/neck squamous carcinomas (11-13). Additionally, UBE2T has been identified to act as an oncogene in numerous types of cancer. For example, UBE2T promotes cell proliferation, invasion and metastasis in nasopharyngeal carcinoma (14), and induces the ubiquitination of p53 in hepatocellular carcinoma, promoting cell growth (15). Elevated expression of UBE2T also serves an oncogenic role in prostate cancer (16). Knockdown of UBE2T suppresses the proliferation and invasion of osteosarcoma and gastric cancer cells $(17,18)$. Furthermore, cell cycle arrest and apoptosis are induced by UBE2T silencing in bladder cancer (19). In breast cancer, UBE2T overexpression results in the degradation of BRCA1 and poor clinical outcome (20). Furthermore, UBE2T is overexpressed in lung cancer and is involved in resistance to chemotherapeutic drugs (21-23). At present, the role of the UBE2T gene in the development and progression of RCC is yet to be investigated.

This study aimed to identify the role of UBE2T in RCC; therefore, the expression of UBE2T was detected in RCC tissues and cells, and its clinical relevance to RCC was analyzed. Furthermore, the effects of UBE2T knockdown on the proliferation, colony formation and invasion of RCC cells were studied in vitro, and in vivo in a nude mouse model. Additionally, the effects of UBE2T knockdown on the phosphorylation of PI3K, Akt and mTOR were investigated via western blot analysis.

\section{Materials and methods}

Clinical samples and ethics statement. A total of 52 fresh surgical tissues and matched adjacent normal tissues from patients (15-62 years old, 36 males and 16 females) diagnosed with RCC were collected from June 2014 to July 2016 at the Department of Urology Surgery of First Affiliated Hospital of Jiamusi University, flash frozen in liquid nitrogen and stored at $-80^{\circ} \mathrm{C}$. Patients that did not receive chemotherapy or radiotherapy prior to surgery were selected for this study. Completed signed clinical information was collected. The pathological stage of patients was established based on the TNM classification system from the WHO (24). Total RNA and protein were extracted and stored at $-80^{\circ} \mathrm{C}$, and used for reverse transcription-quantitative PCR (RT-qPCR) and western blotting, respectively. Patients were separated into high- and low-expression groups for survival analysis based on their levels of UBE2T expression; a fold change $>2$ in expression in tumor tissue compared with in normal tissue was considered high, whereas a fold change $\leq 2$ was considered low.

Written informed consent was obtained from all patients. All experiments were approved by the Institutional Review Board of The First Affiliated Hospital of Jiamusi University.

Cell culture and transfection of small interfering RNA (siRNA). Human renal cancer cell lines (786-O, ACHN and OSRC-2) and a non-cancer cell line (293) were purchased (Cell Bank of the Chinese Academy of Sciences) and cultured in RPMI-1640 medium (Hyclone; GE Healthcare Life Sciences) with $10 \%$ fetal bovine serum (Gibco; Thermo Fisher Scientific, Inc.) in a humidified atmosphere with $5 \% \mathrm{CO}_{2}$ at $37^{\circ} \mathrm{C}$. siRNA fragments targeting human UBE2T (siUBE2T; sequence, 5'-GCAACTGTGTTGACCTCTATT-3') and negative control
(siNC; sequence, 5'-GCTTCGGATACGTTTCCTAAT-3') were synthesized (Shanghai Telebio Biomedical Co., Ltd.) and transfected into $786-\mathrm{O}$ cells $\left(1 \times 10^{5}\right)$ using Lipofectamine ${ }^{\circledR}$ 2000 (Invitrogen; Thermo Fisher Scientific, Inc.). The dosage of siRNA used was $1.0 \mu \mathrm{M}$, and the interval between transfection and subsequent experiments was $6 \mathrm{~h}$.

Construction of a UBE2T overexpression plasmid (oeUBE2T) and transfection. The coding sequence for UBE2T (synthesized by Synbio Technologies) was transferred into vector pcDNA3.1 (Invitrogen; Thermo Fisher Scientific, Inc.) using EcoRI and NotI restriction enzymes. Subsequently, $\sim 1.5 \mu \mathrm{g}$ of the recombined plasmid pcDNA3.1-UBE2T or empty vector pcDNA3.1 were transfected into 786-O cells $\left(1 \times 10^{5}\right)$ using Lipofectamine $^{\circledR} 2000$. After culturing for $48 \mathrm{~h}$, total protein was extracted from the treated cells, stored at $-80^{\circ} \mathrm{C}$ and used for western blotting.

Cell proliferation assay. siUBE2T- or siNC-transfected 786-O cells were seeded into a 96-well plate at 1,000 cells/well. Following incubation for $48 \mathrm{~h}$ at $37^{\circ} \mathrm{C}$, the culture medium was replaced and $20 \mu \mathrm{l}$ MTT reagent $(5 \mathrm{mg} / \mathrm{ml}$; Sigma-Aldrich; Merck KGaA) was added. After a further $4 \mathrm{~h}$ at $37^{\circ} \mathrm{C}$, supernatants were removed and $200 \mu \mathrm{l}$ DMSO was added. The absorbance was detected at a wavelength of $490 \mathrm{~nm}$ using a microplate reader. Wortmannin ( $5 \mathrm{nM}$; Sigma-Aldrich; Merck $\mathrm{KGaA}$ ) was used to treat $786-\mathrm{O}$ cells transfected with siUBE2T, siNC or oeUBE2T for $24 \mathrm{~h}$ at $37^{\circ} \mathrm{C}$.

Colony formation assay. siUBE2T-or siNC-transfected 786-O cells were seeded in a $60-\mathrm{mm}$ dish at 2,000 cells/dish. After 2 weeks at $37^{\circ} \mathrm{C}, 100 \%$ methanol was used to fix the colonies for $2 \mathrm{~min}$ at room temperature. Crystal violet staining solution (Sangon Biotech Co., Ltd.) was used to stain the fixed colonies for $15 \mathrm{~min}$ at room temperature. The number of stained colonies was counted under a Nikon microscope (magnification, x100; Nikon Corporation); five fields were selected randomly for evaluation.

Transwell assay. siUBE2T-or siNC-transfected 786-O cells were seeded into the upper chambers of Transwell inserts (8.0- $\mu \mathrm{m}$ pore size; BD Biosciences) with or without Matrigel matrix at $5 \times 10^{4}$ cells/well in DMEM containing $2 \% \mathrm{FBS}$. DMEM containing $20 \%$ FBS (500 $\mu \mathrm{l})$ was placed in the lower chambers. Following incubation for $48 \mathrm{~h}$ at $37^{\circ} \mathrm{C}$, the cells in the bottom of each chamber were fixed with $100 \%$ methanol for $5 \mathrm{~min}$ at room temperature and stained with $0.1 \%$ crystal violet staining solution for $15 \mathrm{~min}$ at room temperature. Five fields were selected randomly and images were captured. Subsequently, the cell number was counted under a Nikon microscope (magnification, x100; Nikon Corporation).

$R T-q P C R$. Total RNA was extracted from tissues and cell lines using TRIzol ${ }^{\circledR}$ reagent (Invitrogen; Thermo Fisher Scientific, Inc.), and was treated with RNase-free DNase (Promega Corporation). Total RNA (1 $\mu \mathrm{g})$ was used for synthesis of first strand of cDNA using a reverse transcription kit (Takara Biotechnology Co., Ltd.). The protocol used was as follows: $42^{\circ} \mathrm{C}$ for $50 \mathrm{~min}$. qPCR was performed using a Hifair III One Step RT-qPCR SYBR Green kit (Yeasen 
Table I. The primers for RT-qPCR for each gene.

\begin{tabular}{lll}
\hline Gene & \multicolumn{1}{c}{ Forward primer (5'-3') } & \multicolumn{1}{c}{ Reverse primer (5'-3') } \\
\hline UBE2T & CGAGCTCGTAGAAATATTAGGTGGA & TCATCAGGGTTGGGTTCTGAC \\
GAPDH & GAGAAGGCTGGGGCTCATTT & AGTGATGGCATGGACTGTGG
\end{tabular}

UBE2T, ubiquitin-conjugating enzyme E2T.

Biotech Co., Ltd.) using a Stepone Real-time PCR system (Applied Biosystems; Thermo Fisher Scientific, Inc.). qPCR was conducted as follows: $95^{\circ} \mathrm{C}$ for $5 \mathrm{~min}$, then 40 cycles of $95^{\circ} \mathrm{C}$ for $10 \mathrm{sec}$ and $60^{\circ} \mathrm{C}$ for $30 \mathrm{sec}$. GAPDH was used as the internal control. The relative expression of each gene was calculated using the $2^{-\Delta \Delta C q}$ method, in which $\Delta \mathrm{Cq}=\mathrm{Cq}$ (gene of interest)-Cq(GAPDH) (25). The primers used are presented in Table I.

Western blot analysis. Total protein was extracted using a Nuclear and Cytoplasmic Protein Extraction kit (Beyotime Institute of Biotechnology) and quantified via the BCA method using an Enhanced BCA Protein Assay kit (Beyotime Institute of Biotechnology). Proteins (20 $\mu \mathrm{g} /$ lane) were separated via $10 \%$ SDS-PAGE and transferred onto PVDF membranes. Membranes were blocked with 5\% nonfat milk at room temperature for $1 \mathrm{~h}$. Subsequently, the PVDF membranes were incubated with primary antibodies at $4^{\circ} \mathrm{C}$ for $12 \mathrm{~h}$, followed by washing with $0.05 \%$ TBS-Tween- 20 three times. The primary antibodies used during the study were specific for phosphorylated (p)-AKT (1:500; cat. no. ab38449; Abcam), p-PI3K (1:500; cat. no. ab138364; Abcam), p-mTOR (1:2,000; cat. no. ab109268; Abcam), total AKT (1:2,000; cat. no. ab179463; Abcam), total PI3K (1:2,000; cat. no. ab180967; Abcam), total mTOR (1:1,000; cat. no. ab2732; Abcam), UBE2T (1:1,000 cat. no. ab140611; Abcam), fibronectin (1:1,000; cat. no. ab45688; Abcam), E-cadherin (1:600; cat. no. 14472; Cell Signaling Technology, Inc.), N-cadherin (1:1,000; cat. no. 4061; Cell Signaling Technology, Inc.), vimentin (1:500; cat. no. 3932; Cell Signaling Technology, Inc.) and GAPDH (1:1,000; cat. no. 5174; Cell Signaling Technology, Inc.). PVDF membranes were then incubated with secondary antibodies for $1 \mathrm{~h}$ at $37^{\circ} \mathrm{C}$, followed by washing with TBS three times. The secondary antibodies included horseradish peroxidase-conjugated sheep anti-mouse IgG (1:1,000; cat. no. HAF007; R\&D Systems, Inc.) and anti-rabbit IgG (1:1,000; HAF008; R\&D Systems, Inc.). Finally, the membranes were analyzed using an ECL Chemiluminescence Detection kit (Beyotime Institute of Biotechnology). PhotoShop CS6 software was used to quantify protein expression following western blotting (Adobe Systems, Inc.).

In vivo antitumor growth assay. A total of $16 \mathrm{BALB} / \mathrm{c}-$ nu mice (age, 7 weeks; weight, 20-25 g) were obtained from Shanghai SLAC Laboratory Animal Co., Ltd. and were allocated into two groups: siUBE2T and siNC, with 8 mice/group. Animals were maintained at a constant temperature of $25^{\circ} \mathrm{C}$ with $\sim 50 \%$ humidity, under a regular 12:12-h light/dark photoperiod with food and water available ad libitum. Animals were inoculated into the right flank with $3 \times 10^{6}$ cancer cells following transfection for $24 \mathrm{~h}$ with siUBE2T or siNC and monitored every day for 35 days. Tumor volume was calculated twice a week using the following equation: $\mathrm{V}=\left(\mathrm{LxW}^{2}\right) / 2$, where $\mathrm{V}$ is the tumor volume, $\mathrm{L}$ is the length and $\mathrm{W}$ is the width of the tumor. The study was approved by the Animal Research Ethics Committee of The First Affiliated Hospital of Jiamusi University. On day 35, all mice were euthanized according to the protocols approved by the Ethics Committee. All the experiments on mice were performed within the guidelines of the Institutional Animal Care and Use Committee of The First Affiliated Hospital of Jiamusi University.

Statistical analysis. All experiments were repeated at least three times, and the data are presented as the means \pm standard deviation. Differences between the groups were compared using one-way ANOVA followed by Tukey's post hoc test. $\chi^{2}$ test was used to analyze associations between UBE2T expression and the clinicopathological factors of patients with RCC. The Kaplan-Meier method and log-rank test were used to analyze the association between UBE2T expression and the survival of patients with RCC. $\mathrm{P}<0.05$ was considered to indicate a statistically significant difference.

\section{Results}

UBE2T exhibits clinical significance in RCC. To investigate the role of UBE2T in RCC, a total of 52 RCC tissues and adjacent normal tissues were collected, and the expression of UBE2T was determined via RT-qPCR. As presented in Fig. 1A, UBE2T was overexpressed in tumor tissues compared with adjacent normal controls. The mean mRNA expression of UBE2T in tumor tissues was four-fold that in the adjacent controls. Additionally, UBE2T expression was notably increased at the mRNA and protein levels in RCC cell lines compared within 293 cells (Fig. 1B and C). Pathological association analysis revealed that UBE2T expression was associated with TNM grade $(\mathrm{P}=0.001)$ and pathological stage $(\mathrm{P}=0.001)$ in patients with RCC (Table II). Furthermore, Kaplan-Meier analysis indicated that the survival rate of patients with RCC with high UBE2T expression was significantly decreased compared with patients with low UBE2T expression (18.2 vs. $42.1 \%$; $\mathrm{P}=0.01$; Fig. 1D). These data suggested that UBE2T exhibited clinical relevance for RCC and was associated with poor prognosis.

UBE2T regulates cell proliferation and colony formation in RCC. To investigate the function of UBE2T in RCC, 786-O cells were transfected with siUBE2Tor siNC. As 

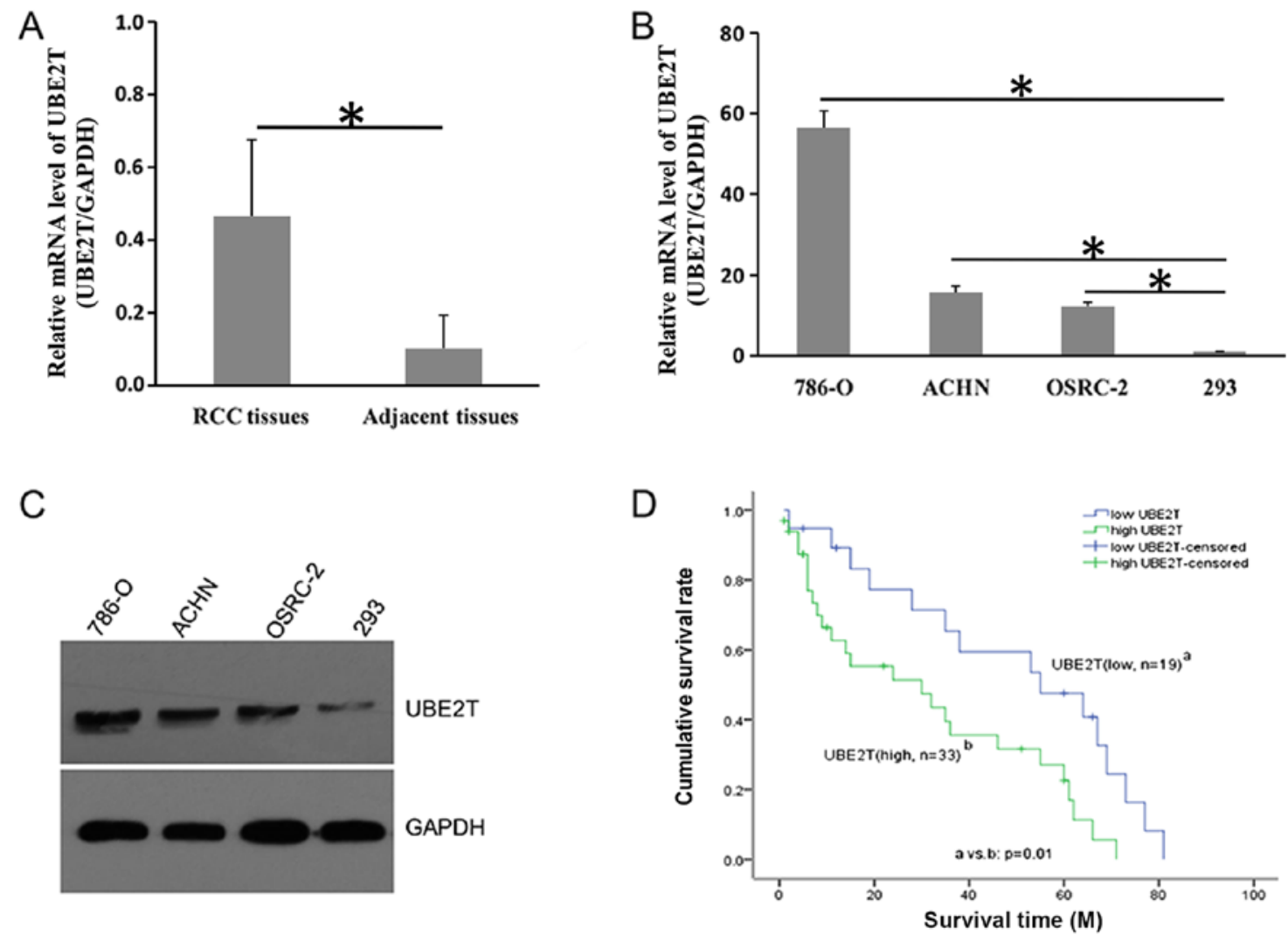

Figure 1. Expression of UBE2T in RCC. UBE2T expression was upregulated in (A) RCC tissues, and in RCC cell lines at the (B) mRNA and (C) protein levels. (D) UBE2T expression was negatively associated with the 5-year survival of patients with RCC. " $\mathrm{P}<0.05$. RCC, renal cell carcinoma; UBE2T, ubiquitin-conjugating enzyme E2T.

Table II. Association of UBE2T expression with clinicopathological factors in renal cell carcinoma.

\begin{tabular}{lccc}
\hline & \multicolumn{2}{c}{ UBE2T expression } & \\
\cline { 2 - 3 } Factor & Low $(\mathrm{n}=19)$ & High $(\mathrm{n}=33)$ & P-value \\
\hline Sex & & & 0.477 \\
Male & 9 & 19 & \\
Female & 10 & 14 & \\
TNM stage & & & 0.001 \\
I/II & 14 & 8 & \\
III/IV & 5 & 25 & \\
Grade & & & 0.001 \\
G1+G2 & 16 & 10 & \\
G3+G4 & 3 & 23 & \\
\hline
\end{tabular}

UBE2T, ubiquitin-conjugating enzyme E2T.

presented in Fig. 2A and B, UBE2T was successfully knocked down at the mRNA and protein level in 786-O cells following siRNA transfection; the knockdown efficiency was $>70 \%$. The proliferation rate of 786-O cells was significantly decreased by $\sim 65 \%$ following knockdown of UBE2T (Fig. 2C). The ability to form colonies was also inhibited; as presented in Fig. 2D and $\mathrm{E}$, the number of siUBE2T-treated 786-O cell colonies was significantly decreased compared with the control. Conversely, the migration and invasion of 786-O cells was not significantly affected by UBE2T (Fig. 3A-D). In conclusion, the results indicated that UBE2T contributed to the proliferation and growth, but not migration of 786-O cells.

UBE2T regulates the expression of epithelial-mesenchymal transition (EMT) markers in RCC. EMT is an important process during the transformation of normal cells into tumor cells (26). Typical molecular markers of epithelial cells are E-cadherin and fibronectin, whereas $\mathrm{N}$-cadherin and vimentin are frequently used markers of mesenchymal cells $(27,28)$. As presented in Fig. 4A and B, the expression of E-cadherin and fibronectin was notably increased in 786-O cells following UBE2T knockdown; conversely, the levels of $\mathrm{N}$-cadherin and vimentin were reduced. The results indicated that UBE2T may participate in the EMT process in RCC.

UBE2T is critical for the activation of PI3K/Akt/mTOR signaling in RCC. PI3K/Akt and the mTOR signaling pathway serve important roles in embryonic development; however, these two signaling pathways are frequently overactivated in tumors $(29,30)$. In the present study, it was demonstrated that the levels of PI3K, Akt and mTOR phosphorylation were markedly decreased following UBE2T knockdown in 786-O cells (Fig. 5A). Conversely, the levels of total PI3K and total AKT were not altered; however, total mTOR expression was notably decreased. Wortmannin, a specific Akt inhibitor, did not further reduce the phosphorylation levels of PI3K, Akt 

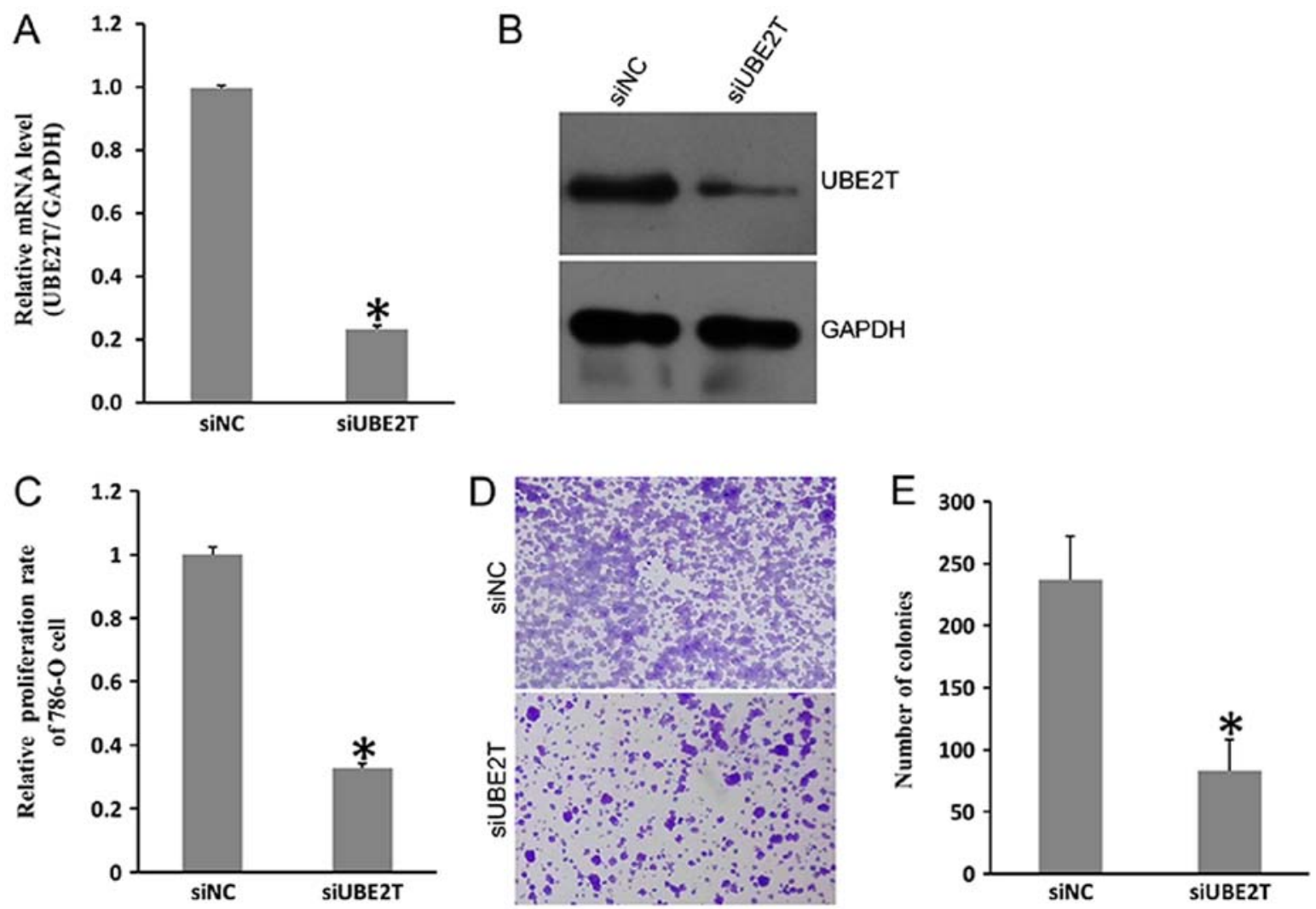

Figure 2. UBE2T knockdown inhibits the proliferation and colony formation of 786-O cells. UBE2T was knocked down at the (A) mRNA level and (B) protein level following transfection with siUBE2T. UBE2T knockdown (C) inhibited the proliferation and (D and E) reduced the colony formation of 786-O cells, as determined via MTT and colony formation assays, respectively. "P<0.05 vs. siNC. NC, negative control; si, small interfering RNA; UBE2T, ubiquitin-conjugating enzyme E2.

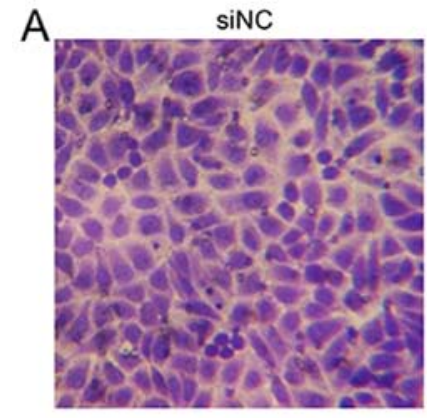

C

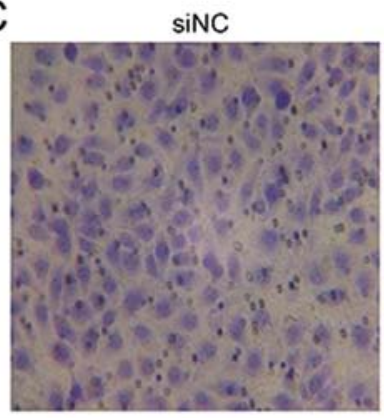

SIUBE2T

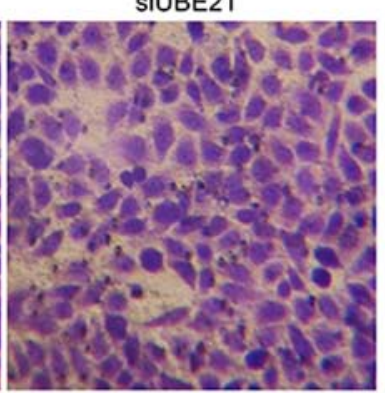

SIUBE2T

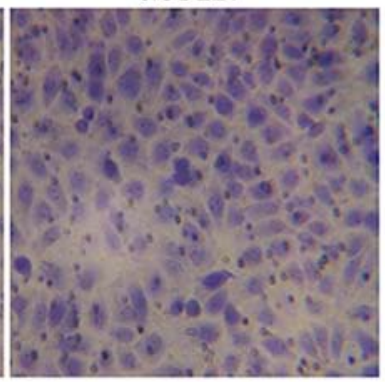

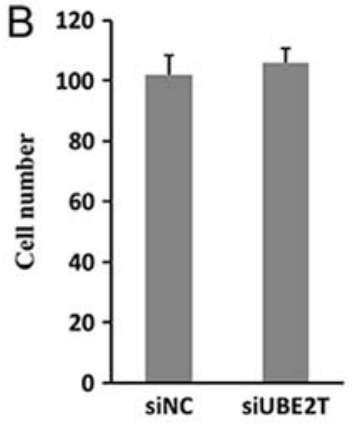

D

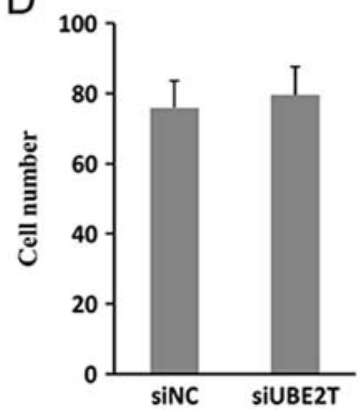

Figure 3. UBE2T does not affect the migration and invasion of 786-O cells. 786-O cells transfected with siUBE2T or siNC did not exhibit significantly different (A and B) migration or (C and D) invasion, as determined using a Matrigel assay (magnification, $x 100)$. NC, negative control; si, small interfering RNA; UBE2T, ubiquitin-conjugating enzyme E2.

and mTOR following UBE2T knockdown. Additionally, the cell proliferation rate in the siUBE2T-transfected group was not significantly different to that in the siUBE2T/wortmannin or siNC/wortmannin groups (Fig. 5B). Conversely, oeUBE2T 

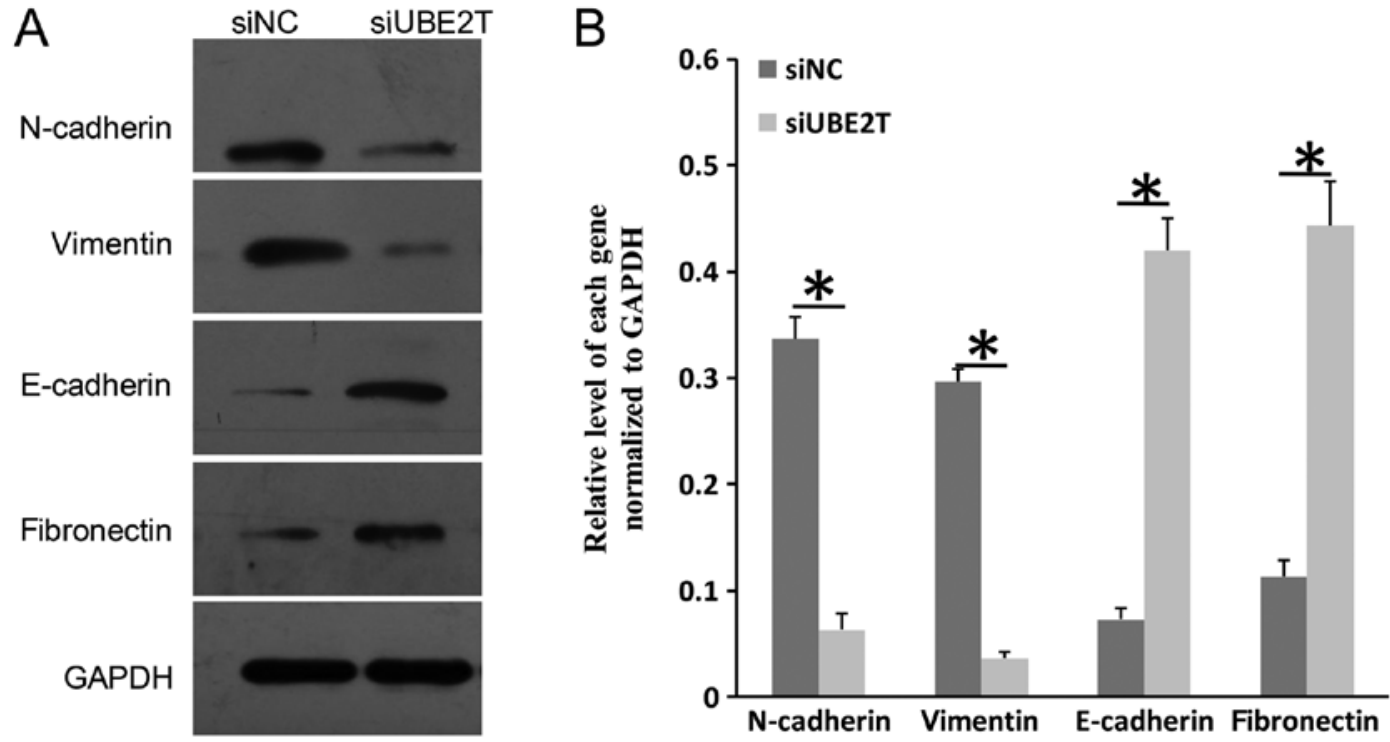

Figure 4. UBE2T knockdown regulates the expression of epithelial-mesenchymal transition markers. (A) Representative western blot demonstrating decreased expression of the mesenchymal cell markers $\mathrm{N}$-cadherin and vimentin, and increased expression of the epithelial markers E-cadherin and fibronectin in siUBE2T-transfected 786-O cells compared with siNC-transfected cells. (B) Expression of each gene was analyzed with PhotoShop CS6 software. "P<0.05. $\mathrm{NC}$, negative control; si, small interfering RNA; UBE2T, ubiquitin-conjugating enzyme E2.
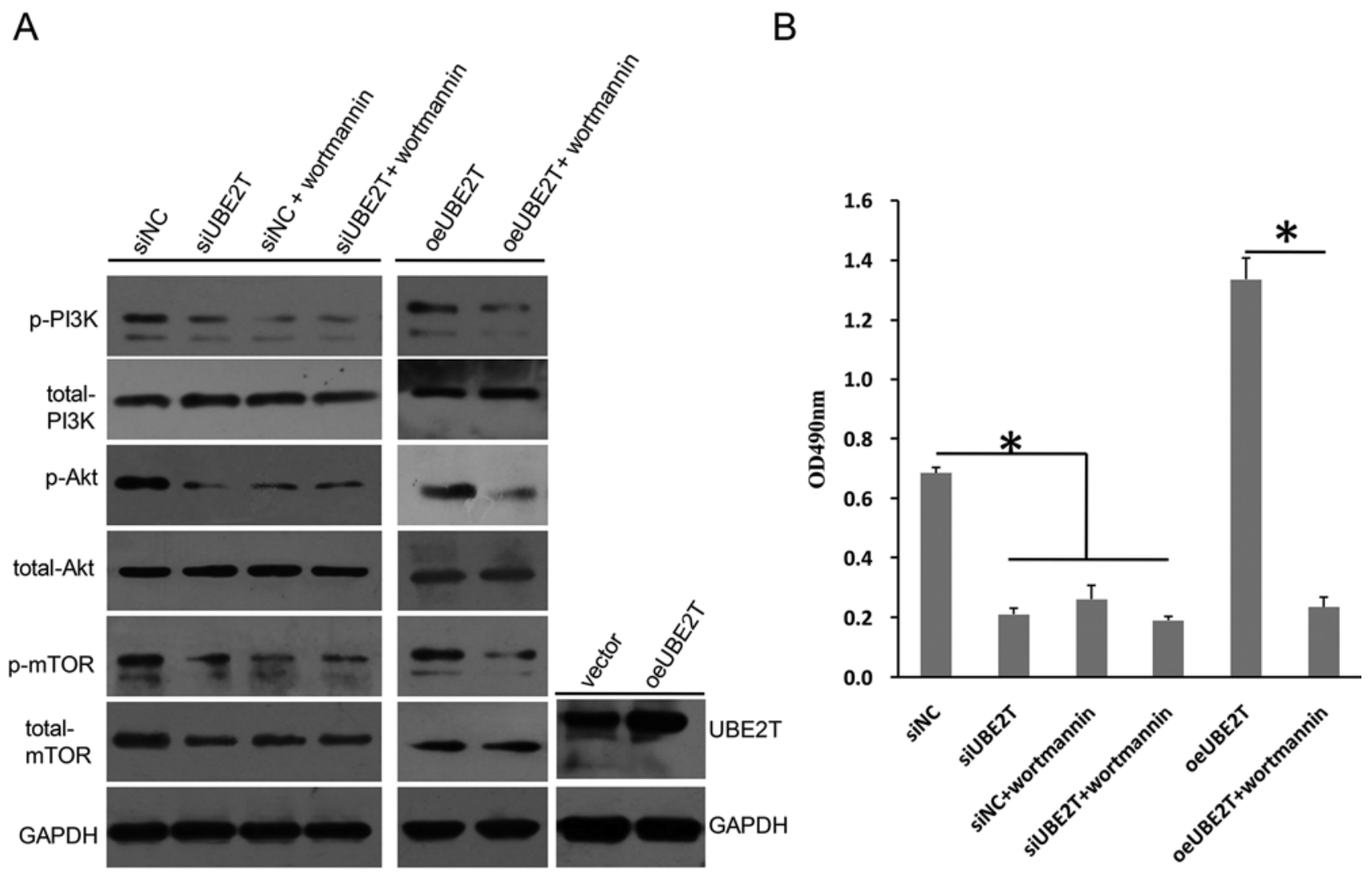

Figure 5. UBE2T knockdown regulates PI3K/AKT/mTOR signaling. (A) Phosphorylation levels of PI3K, Akt and mTOR were decreased in 786-O cells following knockdown of UBE2T, but were increased when UBE2T was overexpressed; the expression of total PI3K or total Akt was not notably altered, whereas total mTOR levels were markedly decreased following UBE2T knockdown. Wortmannin reversed the effects of oeUBE2T. (B) Proliferation of 786-O cells was inhibited by siUBE2T, but enhanced by oeUBE2T in a Wortmannin-sensitive manner. " $\mathrm{P}<0.05$. NC, negative control; oe, overexpression plasmid; p-, phosphorylated; si, small interfering RNA; UBE2T, ubiquitin-conjugating enzyme E2; vector, empty vector.

transfection upregulated the expression of UBE2T compared with the control, and increased the levels of p-PI3K, p-Akt and p-mTOR compared with siNC (Fig. 5A); however, the effects on phosphorylation were reversed by wortmannin, which also significantly decreased cell proliferation even when UBE2T was overexpressed in 786-O cells (Fig. 5B). Therefore, these 

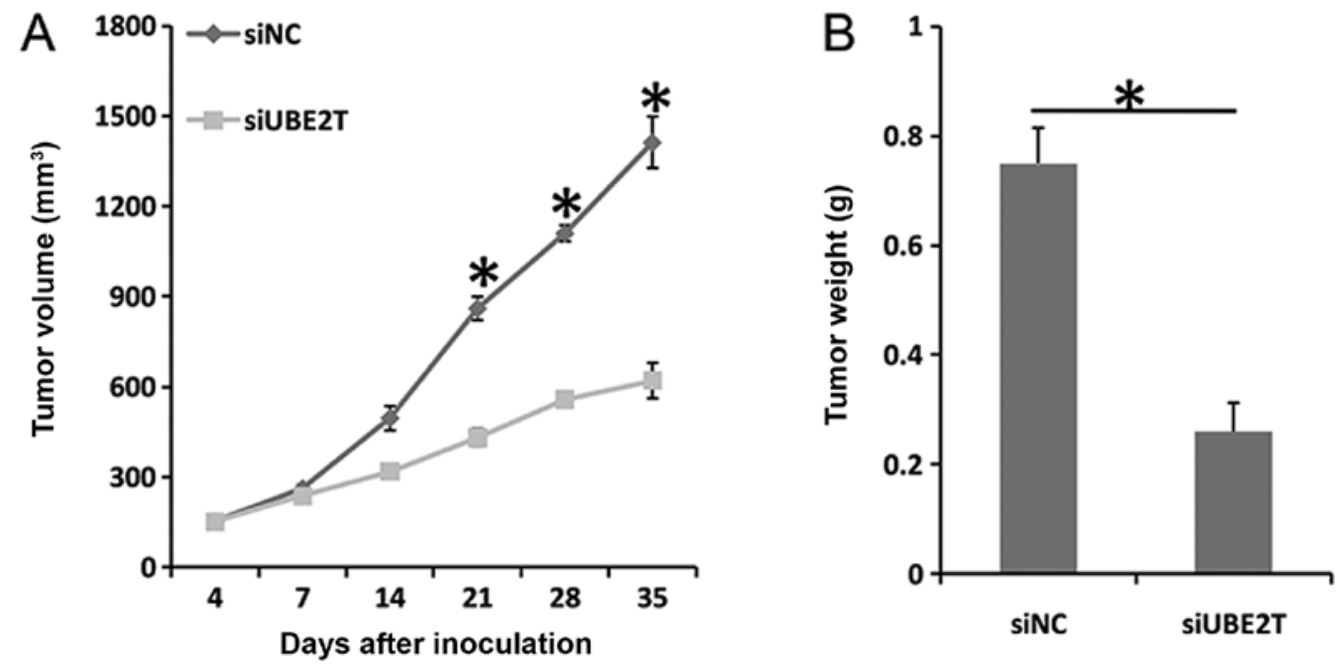

Figure 6. Xenograft RCC tumor growth in mice is suppressed by UBE2T knockdown. (A) Tumor volumes of RCC xenografts derived from siUBE2T-transfected cells were significantly decreased compared with the siNC group " $\mathrm{P}<0.05$ vs. siUBE2T. (B) Tumor weights of RCC xenografts derived from siUBE2T-transfected cells were significantly decreased compared with the siNC group at day 35 . " $\mathrm{P}<0.05$. NC, negative control; RCC, renal cell carcinoma; si, short interfering RNA; UBE2T, ubiquitin-conjugating enzyme E2.

data suggested that UBE2T was involved in regulation of the activation of the PI3K/Akt and mTOR signaling pathways in 786-O cells.

UBE2T knockdown suppresses xenograft tumor growth in vivo. As presented in Fig. 6A, tumor growth was significantly suppressed following UBE2T knockdown. The mean tumor volume in si-UBE2T mice was significantly decreased compared with the control on day $35\left(1,412.5 \mathrm{~mm}^{3}\right.$ vs. $\left.620 \mathrm{~mm}^{3} ; \mathrm{P}<0.05\right)$. In addition, the mean tumor weight was significantly decreased following UBE2T knockdown $(0.26 \mathrm{~g}$ vs. $0.75 \mathrm{~g} ; \mathrm{P}<0.05$; Fig. 6B). Therefore, it was suggested that UBE2T contributed to xenograft tumor growth in vivo.

\section{Discussion}

RCC is a common malignant tumor characterized by poor prognosis, late diagnosis, frequent recurrence and heterogeneity (1-3). The primary therapy for patients with RCC is surgical removal followed by chemotherapy or radiotherapy; however, traditional therapeutics have not improved the quality of life of patients with RCC $(31,32)$. Therefore, there is a requirement for further investigation into RCC. At present, a series of genes have been reported to serve important roles during the tumorigenesis of RCC; however, the critical genes vary between each report $(33,34)$, potentially due to the heterogeneity of RCC.

To the best of our knowledge, the present study is the first to indicate that UBE2T may be a potential candidate predictive factor in RCC. As aforementioned, UBE2T was overexpressed in clinical tumor tissues and was negatively associated with the survival of patients with RCC. The levels of UBE2T were significantly associated with pathological characteristics such as tumor stage and grade. These data suggested the clinical relevance of UBE2T in RCC. This was consistent with a previous study in which UBE2T was reported to be an independent predictive factor in gastric cancer (35). A larger cohort of clinical RCC samples is required to further validate this finding. UBE2T was also reported to be upregulated in tumor cell lines compared with that in control cells. UBE2T knockdown was reported to inhibit cell proliferation and colony formation in 786-O cells in vitro. In vivo, UBE2T contributed to tumor growth. Tumor cells possess the ability to undergo potentially unlimited proliferation (36). Therefore, it was hypothesized that UBE2T served an important role in the progression of RCC. This was consistent with previous findings; for example, previous studies identified that UBE2T overexpression promotes cell proliferation and metastasis in gastric cancer $(18,35)$. Metastasis is another characteristic of cancer (36); however, in the present study, UBE2T expression did not appear to affect the migration and invasion of 786-O cells.

EMT is an important process during the transformation of normal cells to cancer cells, which has been reported to promote the progression of various types of cancer (37). EMT is typically associated with cell migration and invasion in cancer cells; however, in the present study, altered expression of EMT markers was observed in the absence of changes in migration or invasion following siUBE2T transfection. Common molecular markers in EMT include E-cadherin, $\mathrm{N}$-cadherin, vimentin and fibronectin. In the study, the expression of the epithelial markers E-cadherin and fibronectin was upregulated following UBE2T knockdown; whereas the mesenchymal markers $\mathrm{N}$-cadherin and vimentin were downregulated, suggesting that UBE2T regulated the expression of EMT markers. UBE2T may regulate the ubiquitin level of EMT markers in RCC, leading to differential degradation; however, further research is necessary to determine the exact role of UBE2T in EMT in RCC. In conclusion, UBE2T promoted the proliferation but not metastasis of RCC cells, and it may be involved in EMT processes in RCC.

In the present study, it was demonstrated that UBE2T was involved in RCC; however, the mechanisms underlying the effects of UBE2T on cell proliferation in RCC remain unclear. $\mathrm{PI} 3 \mathrm{~K} / \mathrm{Akt}$ is a very important signaling pathway during embryonic development, which frequently induces positive effects on cell growth or proliferation. It has been reported 
to be overactivated in tumors, with increased phosphorylation of PI3K and Akt acting as the critical event (38). In the present study, the phosphorylation levels of PI3K and Akt were increased following UBE2T overexpression and decreased following UBE2T knockdown. These findings suggested that UBE2T may regulate PI3K/Akt signaling in RCC. Wortmannin, a specific inhibitor of Akt, was demonstrated to downregulate the phosphorylation levels of PI3K and Akt even when UBE2T was overexpressed in 786-O cells. The proliferation-promoting role of UBE2T was also suppressed by wortmannin. These data further supported the hypothesis that UBE2T regulated PI3K/Akt signaling in RCC.

$\mathrm{Hu}$ et al reported that UBE2T activated the Akt/glycogen synthase kinase $3 \beta / \beta$-catenin signaling pathway in nasopharyngeal carcinoma (14). UBE2T was also demonstrated to promote cell proliferation via the regulation of PI3K/Akt signaling in osteosarcoma (17). Therefore, it was predicted that UBE2T may activate PI3K/Akt signaling in RCC, as was observed. Additionally, the phosphorylation levels of mTOR were regulated by UBE2T in 786-O cells. mTOR is an important signaling molecule during cell growth (39), which has been demonstrated to crosstalk with PI3K/Akt signaling in cancer cells; for example, PI3K/Akt/mTOR signaling was reported to exhibit positive effects during tumorigenesis in medulloblastoma and thyroid cancer $(38,40)$. Ubiquitin conjugating enzyme E2C (UBE2C) is another member of the ubiquitin-proteasome family that possesses similar functions to UBE2T (41). UBE2C was reported to induce EMT via thePI3K/Akt signaling pathway (42). Activation of PI3K/Akt/mTOR signaling has been revealed to promote EMT in numerous types of cancer (43-45). As aforementioned, UBE2T was observed to be involved in the expression of EMT-associated markers in RCC. Therefore, based on the aforementioned studies and reported findings, it was hypothesized that UBE2T promoted proliferation and EMT in RCC by activating the PI3K/Akt/mTOR signaling pathway; however, further study is required to determine the molecular interactions between UBE2T and the PI3K/Akt/mTOR signal pathway.

In conclusion, to the best of our knowledge, this study is the first to report that UBE2T promoted tumorigenesis in RCC by regulating the $\mathrm{PI} 3 \mathrm{~K} / \mathrm{Akt} / \mathrm{mTOR}$ signaling pathway. Therefore, UBE2T may be a novel target in the treatment of RCC.

\section{Acknowledgements}

Not applicable.

\section{Funding}

No funding was received.

\section{Availability of data and materials}

The datasets used and/or analyzed during the present study are available from the corresponding author on reasonable request.

\section{Authors' contributions}

FHM designed and guaranteed the integrity of the whole study, and reviewed the manuscript. $\mathrm{PH}$ participated in the design of the study and performed the majority of the experiments. BK was involved in the statistical analysis of all data. YPL conducted the study of the literature, participated in the design of major experiments and edited the manuscript. WQH was involved in the collection and analysis of clinical data, and drafted and revised the manuscript.

\section{Ethics approval and consent to participate}

The present study was approved by the Institutional Review Board of First Affiliated Hospital of Jiamusi University and written informed consent was obtained from each patient. Animal experiments were approved by the Animal Research Ethics Committee of The First Affiliated Hospital of Jiamusi University.

\section{Patient consent for publication}

Each patient provided consent for the publication of any data.

\section{Competing interests}

The authors declare that they have no competing interests.

\section{References}

1. Siegel RL, Miller KD and Jemal A: Cancer statistics, 2018. CA Cancer J Clin 68: 7-30, 2018.

2. Rini BI, Campbell SC and Escudier B: Renal cell carcinoma. Lancet 373: 1119-1132, 2009.

3. Bhatt JR and FinelliI A: Landmarks in the diagnosis and treatment of renal cell carcinoma. Nat Rev Urol 11: 517-525, 2014

4. Kamps R, Brandao RD, Bosch BJ, Paulussen AD, Xanthoulea S, Blok MJ and Romano A: Next-generation sequencing in oncology: Genetic diagnosis, risk prediction and cancer classification. Int J Mol Sci 18: E308, 2017.

5. Mirza Z, Schulten HJ, Farsi HM, Al-Maghrabi JA, Gari MA, Chaudhary AG, Abuzenadah AM, Al-Qahtani MH and Karim S: Impact of S100A8 expression on kidney cancer progression and molecular docking studies for kidney cancer therapeutics. Anticancer Res 34: 1873-1884, 2014.

6. Brugarolas J: Molecular genetics of clear-cell renal cell carcinoma. J Clin Oncol 32: 1968-1976, 2014.

7. Alpi AF, Chaugule V and Walden H: Mechanism and disease association of E2-conjugating enzymes: Lessons from UBE2T and UBE2L3. Biochem J 473: 3401-3419, 2016.

8. Machida YJ, Machida Y, Chen Y, Gurtan AM, Kupfer GM, D'Andrea AD and Dutta A: UBE2T is the E2 in the fanconi anemia pathway and undergoes negative autoregulation. Mol Cell 23: 589-596, 2006.

9. Alpi A, Langevin F, Mosedale G, Machida YJ, Dutta A and Patel KJ: UBE2T, the fanconi anemia core complex, and FANCD2 are recruited independently to chromatin: A basis for the regulation of FANCD2 monoubiquitination. Mol Cell Biol 27: 8421-8430, 2007.

10. Richman KA, Lach FP, Abhyankar A, Donovan FX, Sanborn EM, Kennedy JA, Sougnez C, Gabriel SB, Elemento O, Chandrasekharappa SC, et al: Deficiency of UBE2T, the E2 ubiquitin ligase necessary for FANCD2 and FANCI ubiquitination, causes FA-T subtype of fanconi anemia. Cell Rep 12: 35-41, 2015.

11. Mamrak NE, Shimamura A and Howlett NG: Recent discoveries in the molecular pathogenesis of the inherited bone marrow failure syndrome fanconi anemia. Blood Rev 31: 93-99, 2017.

12. Hira A, Yoshida K, Sato K, Okuno Y, Shiraishi Y, Chiba K, Tanaka H, Miyano S, Shimamoto A, Tahara H, et al: Mutations in the gene encoding the E2 conjugating enzyme UBE2T cause fanconi anemia. Am J Hum Genet 96: 1001-1007, 2015.

13. Alter BP: Fanconi anemia and the development of leukemia. Best Pract Res Clin Haematol 27: 214-221, 2014. 
14. Hu W, Xiao L, Cao C, Hua S and Wu D: UBE2T promotes nasopharyngeal carcinoma cell proliferation, invasion, and metastasis by activating the AKT/GSK3//3-catenin pathway. Oncotarget 7: 15161-15172, 2016

15. Liu LP, Yand M, Peng QZ, Li MY, Zhang YS, Guo YH, Chen Y and Bao SY: UBE2T promotes hepatocellular carcinoma cell growth via ubiquitination of p53. Biochem Biophys Res Commun 493: 20-27, 2017

16. Wen M, Kwon Y, Wang Y, Mao JH and Wei G: Elevated expression of UBE2T exhibits oncogenic properties in human prostate cancer. Oncotarget 6: 25226-25239, 2015.

17. Wang Y, Leng H, Chen H, Wang L, Jiang N, Hua X and Yu B: Knockdown of UBE2T inhibits osteosarcoma cell proliferation, migration, and invasion by suppressing the PI3K/Akt signaling pathway. Oncol Res 24: 361-369, 2016.

18. Luo C, Yao Y, Yu Z, Zhou H, Guo L, Zhang J, Cao H, Zhang G, $\mathrm{Li} \mathrm{Y}$ and Jiao Z: UBE2T knockdown inhibits gastric cancer progression. Oncotarget 8: 32639-32654, 2017.

19. Gong YQ, Peng D, Ning XH, Yang XY, Li XS, Zhou LQ and Guo YL: UBE2T silencing suppresses proliferation and induces cell cycle arrest and apoptosis in bladder cancer cells. Oncol Lett 12: 4485-4492, 2016.

20. Ueki T, Park JH, Nishidate T, Kijima K, Hirata K, Nakamura Y and Kataqiri T: Ubiquitination and downregulation of BRCA1 by ubiquitin-conjugating enzyme E2T overexpression in human breast cancer cells. Cancer Res 69: 8752-8760, 2009.

21. Ramaekers CH, van den Beucken T, Meng A, Kassam S, Thoms J, Bristow RG and Wouters BG: Hypoxia disrupts the fanconi anemia pathway and sensitizes cells to chemotherapy through regulation of UBE2T. Radiother Oncol 101: 190-197, 2011.

22. Perez-pena J, Corrales-sanchez V, Amir E, Pandiella A and Ocana A: Ubiquitin-conjugating enzyme E2T (UBE2T) and denticleless protein homolog (DTL) are linked to poor outcome in breast and lung cancers. Sci Rep 7: 17530, 2017.

23. Hao J, Xu A, Xie X, Hao J, Tian T, Gao S, Xiao X and He D Elevated expression of UBE2T in lung cancer tumors and cell lines. Tumour Biol 29: 195-203, 2008.

24. Lopez-Beltran A, Scarpelli M, Montironi R and Kirkali Z: 2004 WHO classification of the renal tumors of the adults. Eur Urol 49 : 798-805, 2006.

25. Livak KJ and Schmittgen TD: Analysis of relative gene expression data using real-time quantitative PCR and the 2(-Delta Delta C(T)) method. Methods 25: 402-408, 2001

26. Chen T, You Y, Jiang H and Wang ZZ: Epithelial-mesenchymal transition (EMT): A biological process in the development, stem cell differentiation, and tumorigenesis. J Cell Physiol 232: 3261-3272, 2017.

27. Figiel S, Vasseur C, Bruyere F, Rozet F, Maheo K and Fromont G: Clinical significance of epithelial-mesenchymal transition markers in prostate cancer. Hum Pathol 61: 26-32, 2017.

28. Scanlon CS, Van Tubergen EA, Inglehart RC and D'Silva NJ: Biomarkers of epithelial-mesenchymal transition in squamous cell carcinoma. J Dent Res 92: 114-121, 2013.

29. Tian T, Li XY and Zhang JH: mTOR signaling in cancer and mTOR inhibitors in solid tumor targeting therapy. Int $\mathbf{J}$ Mol Sci 20: 755, 2019.

30. Faes S and Dormond O: PI3K and AKT: Unfaithful partners in cancer. Int J Mol Sci 16: 21138-21152, 2015.

31. Berquist SW, Yim K, Ryan ST, Patel SH, Eldefrawy A, Cotta BH, Bradshaw AW, Meagher MF, Bindayi A, McKay RR, et al: Systemic therapy in the management of localized and locally advanced renal cell carcinoma: Current state and future perspectives. Int J Urol: Apr 3, 2019 (Epub ahead of print).
32. WiechnoP, Kucharz J, Sadowska M, Michalski W, Sikora-Kupis B, Jonska-Gmyrek J, Poniatowska G, Nietupski K, Ossolinski K and Demkow T: Contemporary treatment of metastatic renal cell carcinoma. Med Oncol 35: 156, 2018.

33. Hsieh JJ, Purdue MP, Signoretti S, Swanton C, Albiges L, Schmidinger M, Heng DY, Larkin J and Ficarra V: Renal cell carcinoma. Nat Rev Dis Primers 3: 17009, 2017.

34. D'Avella C, Abbosh P, Pal SK and Geynisman DM: Mutations in renal cell carcinoma. Urol Oncol: Nov 23, 2018 (Epub ahead of print)

35. Yu H, Xiang P, Pan Q, Huang Y, Xie N and Zhu W: Ubiquitin-conjugating enzyme E2T is an independent prognostic factor and promotes gastric cancer progression. Tumour Biol 37: 11723-11732, 2016

36. Hanahan D and Weinberg RA: Hallmarks of cancer: The next generation. Cell 144: 646-674, 2011.

37. Brabletz T, Kalluri R, Nieto MA and Weinberg RA: EMT in cancer. Nat Rev Cancer 18: 128-134, 2018.

38. Dimitrova V and Arcaro A: Targeting the PI3K/Akt/mTOR signaling pathway in medulloblastoma. Curr Mol Med 15: 82-93, 2015.

39. Cargnello M, Tcherkezian J and Roux PP: The expanding role of mTOR in cancer cell growth and proliferation. Mutagenesis 30: 169-176, 2015.

40. Manfredi GI, Dicitore A, Gaudenzi G, Caraglia M, Persani L and Vitale G: PI3K/Akt/mTOR signaling in medullary thyroid cancer: A promising molecular target for cancer therapy. Endocrine 48: 363-370, 2015

41. Xie C, Powell C, Yao M, Wu J and Dong Q: Ubiquitin-conjugating enzyme E2C: A potential cancer biomarker. Int J Biochem Cell Biol 47: 113-117, 2014.

42. Wang R, Song Y, Liu X, Wang Q, Wang Y, Li L, Kang C and Zhang Q: UBE2C induces EMT through Wnt/Bcatenin and PI3K/Akt signaling pathways by regulating phosphorylation levels of aurora-a. Int J Oncol 50: 1116-1126, 2017.

43. Jin $\mathrm{Y}, \mathrm{Xu} \mathrm{K}$, Chen Q, Wang B, Pan J, Huang S, Wei Y and Ma H: Simvastatin inhibits the development of radioresistant esophageal cancer cells by increasing the radiosensitivity and reversing EMT process via the PTEN-PI3K/AKT pathway. Exp Cell Res 362: 362-369, 2018.

44. Rumman M, Jung KH, Fang Z, Yan HH, Son MK, Kim SJ, Kim J, Park JH, Lim JH, Hong S and Hong SS: HS-173, a novel PI3K inhibitor suppresses EMT and metastasis in pancreatic cancer. Oncotarget 7: 78029-78047, 2016.

45. Zhang GW, Tian X, Li Y, Wang ZQ, Li XD and Zhu CY: Down-regulation of ETS2 inhibits the invasion and metastasis of renal cell carcinoma cells by inducing EMT via the PI3K/Akt signaling pathway. Biomed Pharmacother 104: 119-126, 2018

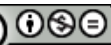

This work is licensed under a Creative Commons Attribution-NonCommercial-NoDerivatives 4.0 International (CC BY-NC-ND 4.0) License. 